\title{
Percutaneous Closure of Atrial Septal Defects. The Role of Transesophageal Echocardiography
}

\section{Simone R. F. Fontes Pedra, Carlos Augusto Cardoso Pedra, Jorge Eduardo Assef, Renata de Sá Cassar, César Augusto Esteves, Sérgio N. Braga, Sérgio Pontes Jr, Valmir Femandes Fontes}

\author{
São Paulo, SP - Brazil
}

\begin{abstract}
Purpose-Evaluation of the role of transesophageal echocardiography in percutaneous closure of atrial septal defects (ASD) with the Amplatzer septal occluder.
\end{abstract}

Methods - Patients were selected for percutaneous closure of ASD by transesophageal echocardiography (TEE), which was also used to monitor the procedure, helping to select the appropriate size of the Amplatzer device, to verify its position, and to access the immediate results of the procedure. During the follow-up, TEE was used to evaluate the presence and magnitude of residual shunt (RS), device position, and right cardiac chamber diameters.

Results - Twenty-two (40\%) of a total of 55 studied patients were selected. Thirteen underwent Amplatzer device implantation, eight are still waiting for it, and one preferred the conventional surgical treatment. All procedures were successful, which was mainly due to proper patient selection. Six (23\%) patients acutely developed $R S$, which spontaneously disapeared at the three-month follow-up examination in three patients. There was a significant reduction in the right ventricle diastolic diameter, from $27 \mathrm{~mm}$ (average) to $24 \mathrm{~mm}$ and $20 \mathrm{~mm}$, one and three months after the procedure, respectively $(p<0.0076)$.

Conclusion - With the aid of TEE, percutaneous closure of ASD can be successfully, safely, and effectively performed.

Key words: atrial septal defects, interventional cardiology, echocardiography

Instituto Dante Pazzanese de Cardiologia - São Paulo

Mailing address: Simone R. F. Fontes Pedra - Instituto Dante Pazzanese de Cardiologia - Av. Dr. Dante Pazzanese, 500 - 04012-180 - São Paulo, SP - Brazil
In recent years, several congenital cardiac defects have been successfully treated with therapeutic catheterization ${ }^{1-10}$. In 1974, King and Mills ${ }^{11}$ made the first attempts at percutaneous closure of atrial septal defects (ASD). Since then, several prostheses have been examined for possible use in this procedure. Currently, five are under investigation with varying results ${ }^{12-19}$. Our experience was developed with the Amplatzer septal occluder ${ }^{20}$, which was experimentally introduced in September $1995^{21}$.

The success of percutaneous closure of ASD is directly related to the proper selection of the patients for implantation. Defects of the ostium secundum type, located in the central portion of the atrial septum, with thick borders and dimensions large enough to sustain the device, are ideal for percutaneous closure. The detailed study of the defect with measurement of its dimensions, in at least two planes, can be performed in a precise way through transesophageal echocardiography (TEE). In addition to patient selection, echocardiography has been used in the hemodynamic room for the continuous monitoring of the procedure, providing additional safety and significantly reducing radiationexposure. Using TEE clinicians not only can verify the position of the Amplatzer septal occluder in relation to the ASD but can also evaluate residual shunt (RS) immediately after can implantation and during the late follow-up. TEE is therefore important in conjunction with this procedure.

In this study our initial experience with percutaneous closure of ASD by means of the Amplatzer septal occluder is presented, stressing the role of TEE.

\section{Methods}

Since August 1997, patients with clinical signs of ASD, diagnosed with transthoracic echocardiography (TTE) as the ostium secundum type, underwent TEE to evaluate the possibility of using percutaneous treatment. Ages of patients ranged from three years and two months to 65 years. TEE was performed after consent of the patient or the patient's guardian. Because only adult esophageal 
probes were available, for the examination to be safely performed it was required that the patient's minimum weight was $16 \mathrm{~kg}$. Patients under 15 years of age underwent TEE with general anesthesia; therefore, an anesthesiologist and a nurse were required to be present in the echocardiography laboratory. Under continuous electrocardiographic monitoring and pulse oximetry, patients were anesthetized with propofol and halothane after receiving midazolam as a preanesthetic. Then, the esophagus was easily lubricated with lidocaine gel and a probe was inserted. At the end of the procedure, the anesthetic effect of midazolam was reversed with flumazenil. Some adults also needed sedation with propofol because they could not tolerate the examination with local anesthesia alone. Ultramark 9 equipment (Advanced Technologies Laboratories Inc., Bothell, Washington, US) was used with an adult biplanar esophageal probe $(11 \mathrm{~mm})$ and color pulsed Doppler. The defect site, position, and number were analyzed, as were the connections of pulmonary veins and cardiac chamber sizes. Hemodynamic impact of the lesion and shunt direction were also noted. Other defects and signs of pulmonary hypertension were also described. The selected patients had defects with borders that had a minimum diameter of 4 to $5 \mathrm{~mm}$ that allowed the satisfactory placement of the occluder's discs in the atrial septum without invading or harming the adjacent cardiac structures. These borders were classified according to their positions as: if in the transversal plane, the one close to the tricuspid valve is the anteroinferior, and the one close to aorta is the anterosuperior; if in the longitudinal plane, the one related to the superior vena cava is the posterosuperior, and the one related to the inferior vena cava is the posteroinferior.

Right ventricle (RV) size prior to implantation was also objectively analyzed, through the measurement of its final diastolic diameter in the $\mathrm{M}$ mode and this was compared to other measurements during follow-up.

The criteria required for implantation were: 1) ostium secundum type of ASD; 2) maximum defect diameter = $21 \mathrm{~mm}$; 3) RV dilatation due to volumetric overload; 4) left-

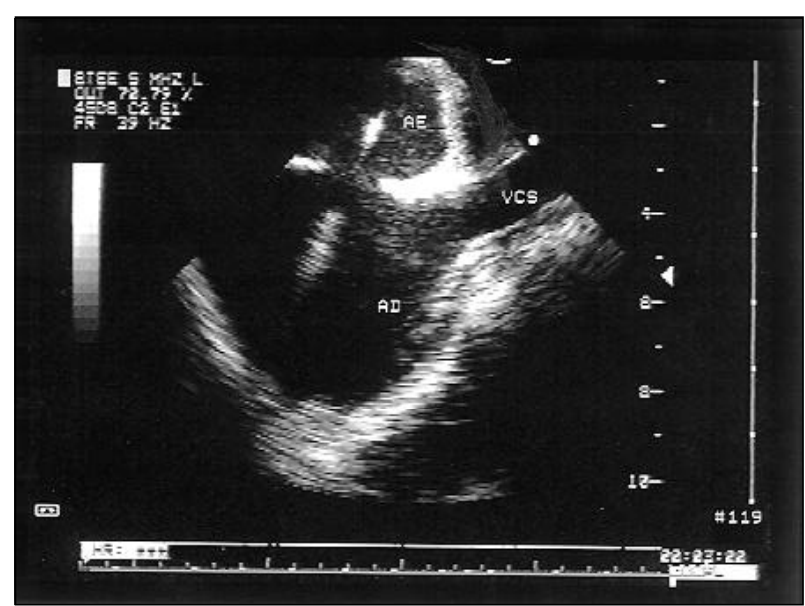

Fig. 1 - Transesophageal echocardiogram of an individual with an ostium secundum type atrial septal defect, presenting favorable characteristics to percutaneous closure with the Amplatzer prosthesis (longitudinal plane). right shunt; 5) minimum distance of 4 to $5 \mathrm{~mm}$ between the defect borders and the coronary sinus, the atrioventricular valves, the right superior pulmonary vein, and the superior and inferior vena cava.

For the selected patients, the percutaneous treatment was offered and performed after they or their guardians had read all the information about the procedure and had signed the consent form.

In the catheterization laboratory, the use of TEE was characterized by: Demonstration of the defect to the interventional team; monitoring and measuring the stretched diameter, monitoring the opening and placement of the discs and waist of the device in the atrial septum; checking the position of the implanted prosthesis prior to the withdrawal of the delivery catheter; and finally, evaluation of RS (location and classification), whenever present.

The stretched diameter was measured with a balloon catheter inflated inside the left atrium and moved towards the atrial septum. After color Doppler showed total occlusion of the defect, the balloon was slowly deflated. At the exact moment it passed through the atrial septum plane, its diameter was obtained by echocardiography. This diameter was compared to that obtained with balloon inflation outside the body, with the same amount of contrast medium used at the moment of passage to the right atrium and estimated using a grid.

RS was characterized by a left-right flow between the defect's borders and the prosthesis, and classified according to the proposal of Boutin and co-workers ${ }^{22}$ : trivial $<1 \mathrm{~mm}$; mild $=1<2 \mathrm{~mm}$; moderate $=2<4 \mathrm{~mm}$; and severe $=4 \mathrm{~mm}$.

After implantation, the patients stayed in the Intensive Care Unit for observation until the following morning, being discharged after chest radiography, electrocardiography (ECG), and TEE were performed. Salicylic acid was prescribed at a dose of 5 to $10 \mathrm{mg} / \mathrm{kg} /$ day for six months, and prophylaxis for infectious endocarditis was indicated for six months or in the presence of RS for life. The proposed protocol for late follow-up consisted of clinical evaluation,

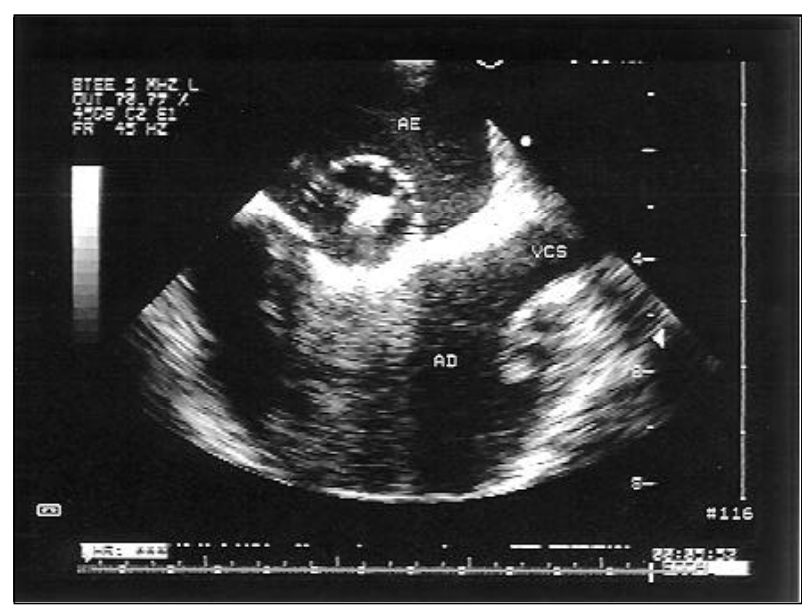

Fig. 2 - Transesophageal echocardiogram (long axis), showing the balloon positioned in the atrial septum during the measurement of the stretched diameter. 
chest radiography, ECG, and echocardiography scheduled for one month, three months (also included a new TEE), and 12 months after implantation.

The non-parametrical test of Friedman was used to compare the average of the final RV diastolic diameters before, one day after, one month after, and three months after implantation. The correlation between the diameter of the ASD and the stretched diameter was evaluated by linear regression.

\section{Results}

Twenty-two of 55 patients studied with TEE were selected for implantation according to the criteria of inclusion. The reasons for excluding the others were: absence of atrial shunt, defects with no hemodynamic impact, defects of the sinus venosus type, with or without partial anomalous pulmonary drainage, multiple defects or defects with dimensions above $21 \mathrm{~mm}$, eccentric ASD, or ASD with very thin borders.

There were no complications during selection, and tracheal intubation was not necessary. The patients were discharged on the same day after a short period of postanesthetic observation.

Thirteen of the 22 selected patients were referred to the catheterization laboratory. One preferred the conventional surgical treatment but eight are still waiting for the procedure. The clinical feature as well as the anatomical and hemodynamic findings of the thirteen patients are listed in table I. Implantation was successfully performed in all patients.

The stretched diameter calculated by TEE was $15 \%$ to $68 \%$ (average of $34 \%$ ) larger than the largest diameter of the ASD determined prior to implantation, also by TEE (Table I). There was a strong linear correlation between them $(\mathrm{r}=0.8646-\mathrm{p}<0.0001)$. The choice of prosthesis was based

\begin{tabular}{|c|c|c|c|c|c|c|c|}
\hline \multicolumn{8}{|c|}{$\begin{array}{l}\text { Table I - Clinical, anatomical and hemodynamic characteristics } \\
\text { of patients who underwent percutaneous closure of atrial } \\
\text { septal defects }\end{array}$} \\
\hline Number & $\begin{array}{c}\text { Age } \\
\text { (years) }\end{array}$ & $\begin{array}{c}\text { Weight } \\
(\mathrm{kg})\end{array}$ & $\begin{array}{l}\text { ASDD } \\
(\mathrm{mm})\end{array}$ & $\mathrm{QP} / \mathrm{QS}$ & $\begin{array}{c}\text { Str. D } \\
(\mathrm{mm})\end{array}$ & Prost. N & Obs. \\
\hline 1 & 5 & 24 & 13.5 & 3.4 & 17 & 19 & - \\
\hline 2 & 13 & 58 & 9.4 & 1.6 & 14 & 16 & \\
\hline 3 & 10 & 27 & 8.7 & 1.5 & 12.3 & 13 & \\
\hline 4 & 7 & 20 & 10.3 & 2.4 & 12.4 & 13 & \\
\hline 5 & 50 & 69 & 20 & 2.6 & 23 & 24 & \\
\hline 6 & 9 & 21 & 13 and 5 & 5.7 & \multicolumn{3}{|c|}{21 and 922 and 8 PCA } \\
\hline 7 & 12 & 27 & 16 & 1.6 & 27 & 24 & \\
\hline 8 & 11 & 31 & 14.8 & 2.8 & 21 & 22 & \\
\hline 9 & 29 & 58 & 21 & 2,1 & 26 & 26 & \\
\hline 10 & 6 & 22 & 16.3 & 2.3 & 20 & 20 & \\
\hline 11 & 21 & 68 & 21 & 2.4 & 25.3 & 26 & \\
\hline 12 & 7 & 21.5 & 15.5 & 2.1 & 18.7 & 19 & \\
\hline 13 & 3 & 20 & 13.5 & 1.6 & 20.6 & 20 & \\
\hline \multicolumn{8}{|c|}{$\begin{array}{l}\text { Where: ASDD - atrial septal defect diameter; Str.D - stretched diameter; } \\
\text { Prost.N - prosthesis number; PDA - persistence of ductus arteriosus; } \\
\text { Obs. - Observation. }\end{array}$} \\
\hline
\end{tabular}

on the diameter of its central point (waist), which was equal to or $1 \mathrm{~mm}$ larger than the stretched diameter obtained.

Patient number 2 presented a very thin posteroinferior border, which impaired its visualization between the two discs of the prosthesis, making it difficult to determine if the occluder's position was correct. Patient number 7 also had very thin borders, with a stretched diameter almost twice the real diameter of the ASD. In this case, the size 24 prosthesis was arbitrarily chosen after confirming that the total extension of the septum would bear the device. This prosthesis fit the septum perfectly and, by the following day the defect was totally occluded. Patient number 6 had two ASD with a border of $8 \mathrm{~mm}$ between the orifices and a short ductus arteriosus. In this case, two prostheses were used. They were arranged like a sandwich (the superior embracing the inferior) in the atrial septum and a Gianturco coil was implanted through the aorta for the occlusion of the ductus arteriosus ${ }^{23}$. During the first prosthesis implantation, the patient experienced a supraventricular tachycardia, immediately reversed with adenosine. At the end of the procedure, the ductus arteriosus was totally occluded and the two prostheses seemed well positioned with no RS. The other procedures were uneventfully performed.

Immediately after the implantation, five patients experienced mild RS. In one of them, RS was only observed by TEE on the following day, probably due to the different incidence of the ultrasound beam of the transthoracic via in relation to the transesophageal one. Similarly, a patient had acute RS that seemed to close on the following day, but the TEE performed three months later again revealed a shunt. In the patient receiving two prostheses, TEE also showed a mild RS $(1.9 \mathrm{~mm})$ three months after implantation. In this case, the left-right flow was at a high speed, and was not noticed immediately after the procedure. Due to these variations, the prevalence of RS was calculated at the third month after implantation. It was $23 \%$ ( 3 patients out of 13 ). There were three spontaneous closures, one occurring 24 hours after the procedure, and the other two by the third month follow-up. In regard to the evaluation of RV dimensions, there was a reduction of some millimeters in the final diasto-

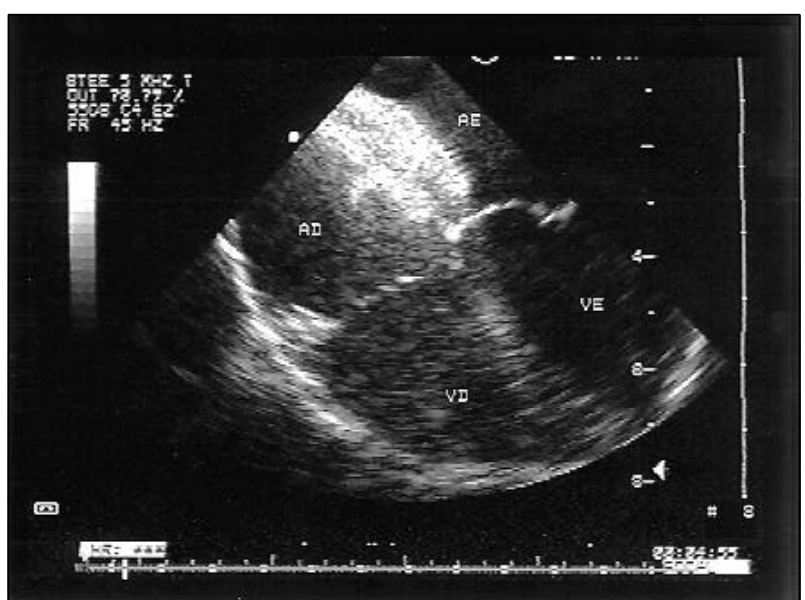

Fig. 3 - Transversal plane showing the perfect position of the device in the atrial septum. 


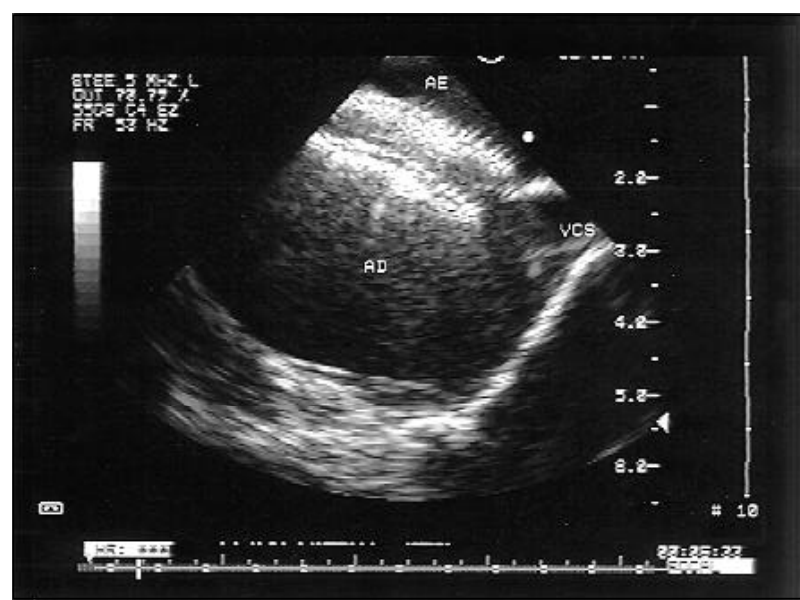

Fig. 4 - Longitudinal axis showing the prosthesis adequately positioned in the atrial septum.

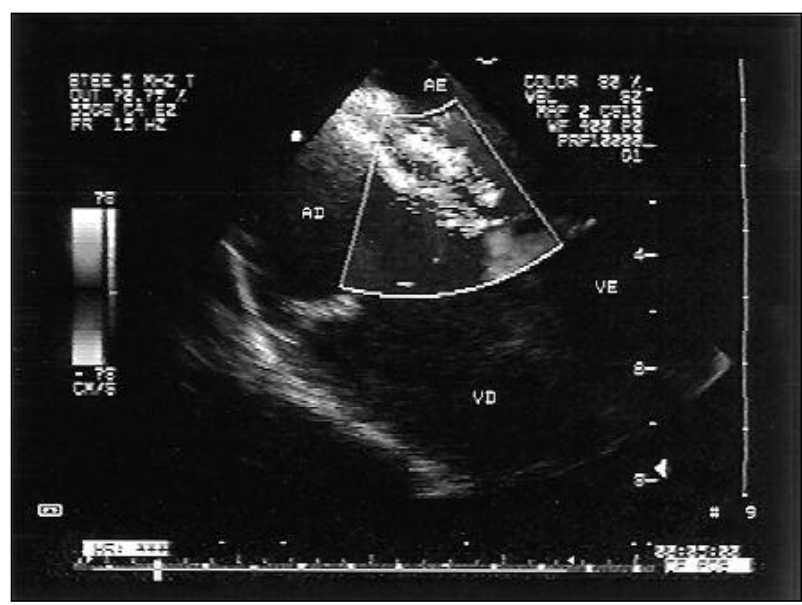

Fig. 5 - Study with color Doppler revealing no residual shunt. LV- left ventricle; RVright ventricle; RA- right atrium; LA- left atrium; SVC- superior vena cava.

lic diameter in all cases. The statistical analysis showed a very significant difference $(\mathrm{p}<0.0076)$ between the averages of the diameters before the implantation, after one and three months. It is necessary, however, to evaluate this result critically, due to the presence of three adults in the sample, which makes the group very heterogeneous.

In our experience, there was neither embolization of the prostheses nor thromboembolic phenomena.

\section{Discussion}

The treatment of ASD by means of therapeutic catheterization can be considered very attractive. The possibility of therapy with no surgical scar, no pain - common in the postoperative period - no transfusion, short hospital stay, and significant reduction in the costs are unequivocal advantages. The devices available for percutaneous closure of ASD have become more technologically refined with time. They are safer, more efficient, have less risk of embolization and of pole fracture, and present a lower incidence of RS. It has also been possible to reduce the profile of the sheaths necessary to deliver the prosthesis ( 6 Fr for the smallest Amplatzer prostheses), increasing the therapeutic possibility for symptomatic infants.

The Amplatzer occluder's basic principle of approaching the ASD is the insertion of its central point in the defect, providing stability to the device and eliminating the need for metal arms or frameworks. This waist centralizes the prosthesis automatically in relation to the septum, independent of the implantation angle, facilitating its positioning. As the discs do not need large dimensions for the device's stabilization, their implantation can be potentially extended to defects not having extensive borders that would keep them apart from other intracardiac structures. These discs as well as the whole metallic structure of nitinol are extremely flexible and do not contain any sharp elements or hooks, which minimizes the risk of perforation during and after the procedure.

As previously reported, the success of implantation is directly related to the proper selection of patients, which was very well demonstrated in our series - all patients referred to percutaneous closure of ASD had a successful outcome. TEE allows perfect visualization posterior structures of the heart, particularly the detailed study of the atrial septum and its defects ${ }^{22}$. For this reason, a transthoracic evaluation suggesting ASD susceptible to percutaneous treatment should be complemented with a TEE. The fact that only $40 \%$ of the 55 patients studied by TEE presented favorable characteristics for this treatment stresses even more the need for selection of patients using this technique. Despite the necessity of general anesthesia for pediatric patients, the procedure can be safely performed on an outpatient basis, as long as equipment for cardiorespiratory monitoring and appropriate anesthetic drugs are readily available. In our experience, patient selection was uneventful.

The possibility of monitoring the implantation with TEE makes the procedure easier because ASD cannot be as well analyzed by angiography. Besides, the technique allows patient and staff to be spared from exposure to radiation ${ }^{24}$. Fifteen minutes of fluoroscopy are necessary for the implantation. With TEE, the use of contrast medium is limited to the initial diagnostic angiography. The Amplatzer septal occluder causes no reverberation of the ultrasound beam; therefore it can be very well evaluated by echocardiography. The verification of the borders between the two discs of the device in the longitudinal and transversal planes provides accurate confirmation that the device is properly positioned. This is a key role of TEE because the prosthesis can only be released when its appropriate position is confirmed.

RS is best evaluated by color Doppler. Angiography immediately after the implantation can reveal a left-right shunt through the prosthesis mesh, since the epithelization process has not yet begun. Through the use of TEE it is possible to evaluate the real presence of RS and to determine the shunt's magnitude by measuring its width ${ }^{22}$. RS rates vary according to the different techniques and prostheses used for evaluation. In a multicenter study of the Sideris prosthesis, RS prevalence in one month was $80.5 \%$, 
dropping to $45 \%$ in six months, and to $19 \%$ in one year ${ }^{13}$. In another study carried out in Toronto with the Clamshell prosthesis (Bard USCI Division, C.R. Bard, Billerica, MA, USA), an incidence of acute RS of $91 \%$ was reported, with regression to $71 \% 24$ hours later. In an average follow-up of 10 months, the rate dropped to $47 \%{ }^{22}$. In the initial global experience with 230 cases of Amplatzer prosthesis implantation, the occlusion rate was higher than $95 \%$ after the first month of follow-up ${ }^{21}$. In our initial experience, there were three patients out of 13 (23\%) with mild RS three months after implantation confirmed by TEE. Maybe our results, in regard to RS, are not so good due to the fact that ours is a small series, and also to the unusual case where two prostheses were simultaneously implanted in the same patient. Sometimes, RS could not be seen by TTE, which is not surprising considering the greater sensitivity of TEE for the visualization of the atrial septum. However, it surprised us to observe RS by TTE, on the following morning, because it had not been visualized by TEE immediately after the implantation in the catheterization laboratory. Maybe the difference in the direction of the ultrasound beam in the two procedures could explain this phenomenon, allowing us to suggest that, after the end of the implantation, RS investigation should also be carried out using TTE.

Trivial and discrete RSs do not cause any volume overload to the right chambers and, many times, do not have murmurs. The disappearance of the fixed splitting of the second cardiac sound can also be observed. Therefore, the ASD can be considered treated from the clinical-functional point of view, even in the presence of these small shunts. It is worth stressing that the real incidence of RS in the postoperative period of ASD correction is not completely known, because there are no consistent studies in this area. The major problem of RS rests in the unknown incidence of infectious endocarditis, especially after the introduction of a foreign body into the septum. This is the reason why prophylaxis is recommended. In our protocol, those patients with a persistent $R S$ after the third month follow-up should have TEE repeated one year after the implantation to determine the necessity of continued prophylaxis.

In conclusion, percutaneous closure of ASD today is a reality. The Amplatzer septal occluder has produced very favorable results with low risks. TEE is fundamental in the selection of candidates for implantation, procedure monitoring, and follow-up of these patients.

\section{Acknowledgments}

We wish to express our gratitude to Mrs. Angela Paes for her help with the statistical analysis, and to the staff of the echocardiography and catheterization laboratories of Instituto Dante Pazzanese de Cardiologia, for the collaboration .

\section{References}

1. Kan JS, White RI, Mitchell SE, Anderson JH, Gardner TJ - Percutaneous transluminal balloon valvuloplasty for pulmonary valve stenosis. Circulation 1984; 69: 554-60.

2. Mrantz PM, Huhta JC, Mullins CE, Murphy DJ, Nihill MR, Ludomirsky A - Results of balloon valvuloplasty in typical and dysplastic pulmonary valve stenosis: Doppler echocardiographic follow-up. J Am Coll Cardiol 1988; 12: 476-91.

3. Rao OS - Balloon pulmonary valvuloplasty: a review. Clinical Cardiology 1989; 12: 55-74.

4. Choy M, Beekman RH, Rocchini AP, et al-Percutaneous balloon valvuloplasty for valvar aortic stenosis in infants and children. Am J Cardiol 1987; 59: 1010-13.

5. Lababidi Z, Wu J, Walls JT - Percutaneous balloon aortic valvuloplasty: results in 23 patients. Am J Cardiol 1984; 53: 194-7.

6. Beekman RH, Rocchini AP, Dick M, et al - Percutaneous balloon angioplasty for native coarctation of the aorta. J Am Coll Cardiol 1987; 10: 1078-84.

7. Delezo JS, Sancho M, Pan M, Romero M, Oliveira C, Luque M-Angiographic follow-up after balloon angioplasty for coarctation of the aorta. J Am Coll Cardiol 1989; 13: 689-95.

8. Kan JS, White RI, Mitchell SE, Farmalett EJ, Donahoo JS, Gardner TJ-Treatment of restenosis of coarctation by transluminal angioplasty. Circulation 1983; 68: 1087-94.

9. Rashkind WJ, Mullins CE, Hellenbrand WE, Tait MA - Nonsurgical closure of patent ductus arteriosus: clinical application of the Rashkind PDA occluder system. Circulation 1987; 75: 583-92.

10. Hellenbrand WE, Mullins CE - Catheter closure of congenital cardiac defects. Cardiol Clinics 1989; 7: 351-68.

11. King DT, Mills NL - Secundum atrial septal defects: non-operative closure during cardiac catheterization. JAMA 1976; 235: 2506-9.

12. Rao PS, Sideris EB, Hausdorf G, et al - International experience with secundum atrial septal defect occlusion by the buttoned device. Am Heart J 1994; 128: 1022-35.

13. Haddad J, Seches A, Finzi L, et al - Oclusão percutânea transvenosa da comunicação interatrial mediante a utilização do buttoned device. Arq Bras Cardiol 1996; 67: 17-22.
14. Das GS, Voss G, Jarvis G, Wyche K, Gunther R, Wilson RF-Experimental atrial septal defect closure with a new transcatheter self-centering device. Circulation 1993; 88 (part I): 1754-64.

15. Sievert H, Babic UU, Ensslen R, et al - Verschluss des vorhofseptumdefektes mit einem neuen Okklisionssystem. Z Kardiol 1996; 85: 97-103.

16. CardioSeal Septal Occlusion System - Nitinol Medical Technologies, Inc. USA Brochure, 1997.

17. Sharafundin MJA, Gu X, Titus J, et al - Transvenous closure of secundum septal defects: preliminary results with a new self-expanding nitinol prosthesis in a swine model. Circulation 1997; 95: 2162-8.

18. Bjornstad PG, Smevik P, Fiane AE, et al - Catheter-based closure of atrial septal defects with a newly developed nitinol double disc: an experimental study. Cardiol Young 1997; 7: 220-4.

19. Bjornstad PG, Masura J, Thaulow E, et al - Interventional closure of atrial septal defects with the Amplatzer device: first clinical experience. Cardiol Young 1997; 7: 277-83.

20. Fontes VF, Pedra CAC, Pedra SRFF, et al - Experiência inicial no fechamento percutâneo da comunicação interatrial com prótese de Amplatzer. Arq Bras Cardiol 1998; 70: 147-53

21. Masura J, Gavora P, Formanek A et al - Transcatheter closure of secundum atrial septal defects using the new self-centering Amplatzer Septal Occluder. Initial Human Experience. Cath Cardiovas Diagn 1997; 42: 388-93.

22. Boutin C, Musewe NN, Smalhorn JF, Dyck JD, Kobayashi T, Benson LN Echocardiographic follow-up of atrial septal defect after catheter closure by double-umbrella device. Circulation 1993; 88: 621-7.

23. PedraCAC, PedraSRFF, Esteves CA, AssefJE, Fontes VF, HijaziZM-Multiple atrial septal defects and patent ductus arteriosus: successful outcome using two Amplatzer septal occluders and Gianturco coils. Cath Cardiovas Diagn 1998; 45: 257-9.

24. Velde VME, Perry ST, Sanders SP - Transesophageal echocardiography with color Doppler during interventional catheterization. Echocardiography 1991; 8: 721-30 